\title{
Burden of Disease and Current Management of Dementia with Lewy Bodies: A Literature Review
}

\author{
Amir Abbas Tahami Monfared · Genevieve Meier • Richard Perry • \\ Darren Joe
}

Received: June 4, 2019 / Published online: September 12, 2019

(c) The Author(s) 2019

\begin{abstract}
Introduction: A significant proportion of dementia is concretely estimated to be attributable to dementia with Lewy bodies (DLB)-one of the most common types of progressive dementia; however, there is a paucity of literature on this disease. We aimed to examine available evidence to gain a better understanding of its treatment landscape, clinical management, and disease burden.
\end{abstract}

Methods: A systematic literature review captured any DLB studies that report on randomised controlled trials (RCTs), epidemiology, disease progression, and economic data. An additional targeted literature review captured studies reporting on clinical management and

Enhanced digital features To view enhanced digital features for this article go to https://doi.org/10.6084/ m9.figshare.9741479.

Electronic supplementary material The online version of this article (https://doi.org/10.1007/s40120019-00154-7) contains supplementary material, which is available to authorized users.

A. A. Tahami Monfared $(\bowtie) \cdot$ G. Meier

Eisai Inc., Woodcliff Lake, USA

e-mail: amir_tahami@eisai.com

\section{A. A. Tahami Monfared}

Epidemiology, Biostatistics, and Occupational

Health, McGill University, Montreal, Canada

R. Perry · D. Joe

Adelphi Values, Bollington, UK quality of life (QoL) in this disease. Publication date was limited to 1 January 2007-26 March 2018, with the exception for RCTs, where no time restrictions were applied.

Findings: Of the 3486 studies initially identified, 55 studies were eligible for inclusion. The studies were mainly from Europe $(n=29)$, the USA $(n=9)$, and Japan $(n=8)$. Mini-Mental State Examination and Neuropsychiatric Inventory scores were the most commonly reported clinical outcomes in RCTs $(n=14)$. The most frequently identified interventions reported in RCTs were donepezil and memantine. Patients with DLB typically reported worse outcomes in relation to efficacy and safety, cognitive impairment, survival, and QoL compared with those with Alzheimer's disease (AD). Additionally, patients with DLB were associated with higher hospitalisation rates and cost of care. Furthermore, there is a reliance on a small number of consensus guidelines. Of these, only one set of guidelines (DLB Consortium) was developed specifically for DLB.

Conclusion: The paucity of data indicates an unmet need in this therapy area. Although several studies look into the clinical and pathological aspects of DLB, consensus guidelines and studies on healthcare utilisation in patients with dementia have largely focused on AD. Additionally, most of the findings were made in comparison with $\mathrm{AD}$.

Funding: Eisai Inc. 
Keywords: Burden of disease; Clinical management; Dementia; Dementia with Lewy bodies; Healthcare resource utilisation; Healthcare costs; Lewy body dementia; Quality of life

\section{INTRODUCTION}

Dementia with Lewy bodies (DLB) is the second most common neurodegenerative dementia in older adults [1-3]. Features of DLB include progressive cognitive decline, visual hallucinations, cognitive "fluctuations" in alertness and attention, autonomic dysfunction, delusions, and depression [1-3]. DLB also features motor signs of Parkinson's disease (PD), such as rigidity, difficulty walking, slowness of movement, and postural instability [1-3].

In 1912, Fritz Jakob Heinrich Lewy (1885-1950) first described eosinophilic intraneuronal inclusions, now known as Lewy bodies, while studying the neuropathology of PD at Alois Alzheimer's laboratory (Munich, Germany) [4]. Half a century later, the neuropathologist John Woodard reported neuropsychiatric symptoms and cognitive decline in 27 patients with autopsy-proven Lewy body disease, of which only about a quarter exhibited parkinsonian symptoms [5]. Over the following decades, Kenji Kosaka (Yokohama City University) and others from Japan reported detailed autopsies of over 20 patients with variable distribution of Lewy bodies in their brain stem and cerebral cortex [6]. These patients presented with varying amounts of cognitive impairment, and neuropsychiatric and motor symptoms [6].

The first international workshop on the disease in 1995 proposed the term "dementia with Lewy bodies". Diagnostic criteria for DLB based on the three core features of fluctuation in cognitive function, visual hallucinations, and spontaneous motor features of parkinsonism were published in 1996 [7]. Multiple revisions of these criteria have been published since then.

DLB may be misdiagnosed as other forms of dementia due to similar clinical and pathological features [8]. For instance, the cognitive domains of DLB and Parkinson's disease dementia (PDD) overlap, e.g. progressive executive impairment, visual-spatial abnormalities, and diminished memory [9]. A misdiagnosis or a delayed diagnosis limits healthcare providers' ability to maximise therapeutic outcomes and improve patient and caregiver quality of life (QoL), thereby adding to disease burden [10].

DLB is an age-related disease, although onset before age 65 years is not uncommon, and is more prevalent in men than women [11]. As of 2013, the incidence of DLB across all ages was 3.9 per 100,000 person-years in the United States (US). This incidence increases to 31.6 per 100,000 person-years in individuals older than 65 years of age [12]. The exact cause of DLB is unknown; the hallmark pathology associated with disease onset is the accumulation of aggregated neuronal proteins called alphasynuclein ( $\alpha$-synuclein), resulting in interference of synaptic transmissions $[3,11]$.

The management of symptoms for DLB includes the use of anxiolytics, antidepressants, melatonin, and other sleep medications [13]. Autonomic symptoms are also often treated with non-pharmacological treatments [13]. However, treatment is largely based on expert opinion.

Despite DLB being the second most common neurodegenerative dementia, research in this disease is not as well advanced as for Alzheimer's disease (AD). In this review, we therefore aim to summarise the available evidence in order to gain a better understating of the treatment landscape, clinical management, and burden of disease associated with DLB.

\section{METHODS}

\section{Search Strategy and Selection Criteria}

Two separate systematic literature reviews were conducted. The first focused on identifying publications reporting on disease progression and economic burden. The second captured randomised controlled trials (RCT) reporting on pharmacological interventions in DLB. An additional targeted literature review captured studies reporting on clinical management and QoL. The search terms used in each search 
strategy utilised a combination of free text searching and "subject headings" in order to ensure that the most relevant literature was identified (see Tables S1, S2, and S3 in the electronic supplementary materials for details). For each search strategy, articles dating from 1 January 2007 to 26 March 2018 were identified through electronic searches via Ovid (Medline, EMBASE, EBM, PsycINFO, and EconLit); an exception was applied for RCTs whereby no time restrictions were applied. We also manually searched reference lists of relevant publications and trial registries for further studies. Included publications were restricted to those written in English and studies which included adult populations.

\section{Data Analysis}

A total of 55 original publications were identified and taken forward for qualitative synthesis, from which information was reviewed and analysed descriptively. Data sharing is not applicable to this article, as no data sets were generated or analysed during the current study. For all publications reporting on RCTs, the validity of individual trials was assessed using the risk-of-bias instrument endorsed by the Cochrane Collaboration [14]. Additionally, within-study bias in RCTs was assessed qualitatively (i.e. either low risk of bias, unclear risk of bias, or high risk of bias among key domains) as per Cochrane guidance [14]. For each study, the bias assessment was conducted independently by two authors.

\section{Ethical Considerations}

This article is based on previously conducted studies and does not contain any studies with human participants or animals performed by any of the authors.

\section{RESULTS}

\section{RCTs}

Overall, 14 RCTs (or extensions of RCTs) reporting on efficacy and safety outcomes in patients with DLB were identified (please see Table S4 in the electronic supplementary material for details) [15-28]. Across these studies, the most commonly identified medications were memantine and donepezil, which were assessed in five and four studies, respectively. All five studies which assessed memantine were conducted in European countries [15-19], and of these, three studies comprised patients from the United Kingdom (UK), Norway, and Sweden $[15,18,19]$, and one consisted of patients from Sweden only [17]. The remaining study from Europe included patients from Austria, France, Germany, the UK, Greece, Italy, Spain, and Turkey [16]. All four donepezil studies were from Japan [20-23]. Additional intervention(s) of interest reported in the identified studies included citalopram, olanzapine, quetiapine, risperidone, and rivastigmine.

An overview of the baseline characteristics of patients in the identified studies is presented in Table S5 in the electronic supplementary material. Five studies included only patients with DLB [20-24] and five included patients with DLB or PDD [15-19]. Of the five studies which included DLB and PDD patients, four were pooled analyses [15, 17-19]. Additionally, one study included patients with DLB or AD [25], one included patients with "probable" DLB [26], and one included patients with DLB, PD and dementia, or $\mathrm{AD}$ [27], the latter of which was a pooled analysis.

The most commonly reported efficacy outcomes in the identified studies included the Mini-Mental State Examination (MMSE) $[15,21-23,25,27,28]$ and variations of the Neuropsychiatric Inventory (NPI) instrument $[15,16,21-24,28]$.

Within these studies there is wide variation in study and demographic characteristics. The degree of heterogeneity across the studies meant that the trials were not directly comparable.

\section{Disease Course or Progression}

\section{Cognitive Decline}

We identified five publications describing cognitive decline in patients with DLB [29-33]. All 
Table 1 Overview of consensus guidelines for the clinical management of patients with DLB

\begin{tabular}{lll}
\hline Organisation & Region & Reference details \\
\hline BAP [45] & UK & O'Brien et al. Clinical practice with anti-dementia drugs: a revised \\
& & (third) consensus statement from the British Association for \\
& & Psychopharmacology. Journal of Psychopharmacology. \\
& & $2017 ; 31(2): 147-168$
\end{tabular}

Produced by the authors for this publication based on data presented in McKeith et al. [46]

$B A P$ British Association for Psychopharmacology, DLB dementia with Lewy bodies, DLBC Dementia with Lewy Bodies Consortium, EFNS-ENS European Federation of Neurological Societies-European Neurological Society, UK United Kingdom

five publications also described cognitive decline in patients with $\mathrm{AD}$, while two publications reported on patients with PDD; [31, 33] one study also included information on patients with vascular dementia (VaD), behavioural variant frontotemporal dementia (bvFTD), and language variant frontotemporal dementia (lvFTD) [32].

Of these five studies, four described the rate of cognitive decline [29-31, 33]. A high level of variance was evident in the outcomes measured and the statistical significance of results between studies. Generally, data from the literature demonstrated that patients with DLB reported worse outcomes in relation to the rate of cognitive decline compared with other types of dementia, particularly AD (Table S6).

One study from the Netherlands investigated the patterns of cognitive decline via linear mixed models in patients with DLB, AD, VaD, bvFTD, and lvFTD [32]. Patients were assessed in five cognitive domains: memory, language, attention, executive and visuospatial functioning, and global cognition (MMSE) [32]. In general, patients with dementia performed worse than controls at baseline in all cognitive domains except visuospatial functioning, which was only impaired in patients with AD or DLB (Table S7) [32]. Patients with DLB showed decline in every cognitive domain except language and global cognition [32].

Three studies described potential predictors of cognitive decline in patients with DLB $[29,31,33]$. Predictors of note indicating increased rates of decline included lower MMSE (B: 0.216, $p<0.009$ ) and higher Unified Parkinson's Disease Rating Scale-part III scores (UPDRS III; $B:-0.063, p<0.03$ ) at baseline [33]. Additionally, patients diagnosed with DLB exhibited a more rapid annual rate of decline than those with AD or PDD when the baseline MMSE score was included as a cofactor in linear mixed-model analyses (Table S6) [31]. Reported factors associated with shorter time to severe dementia [defined as Clinical Dementia Rating score of $3(\mathrm{CDR}=3)$ ] or death according to Cox regression analysis included longer symptom duration [adjusted hazard ratio (HR) 1.15 (1.01, 1.29), $p=0.03$ ], higher CDR global scores [adjusted HR $2.42(1.26,4.65), p=0.008]$, and DLB diagnosis [vs AD; adjusted HR 2.04 (1.12, 3.72), $p=0.02]$ [29].

Additionally, one study reported on the transition of patients from mild cognitive impairment (MCI) to DLB [34]. Among a cohort of amnestic and non-amnestic MCI $(n=327)$ followed annually for up to 12 years, a history of probable rapid eye movement sleep behaviour disorder was found in $80 \%$ of those with 
MCI who developed probable DLB, versus $8 \%$ in those who developed probable AD [34]. Patients with MCI who developed DLB were also more likely to have baseline fluctuations, daytime sleepiness, and subtle, but measurable, extrapyramidal signs [34].

Furthermore, among patients with amnestic MCI $(n=278)$, the rate of progression to probable $\mathrm{AD}$ was $17 \%$ [ 17 per 100 person-years, $95 \%$ confidence interval (CI) 14.9-19.4], and to DLB was $1.5 \%$ (1.5 per 100 person-years, 95\% CI 1.0-2.2) [34]. In contrast, among patients with non-amnestic MCI $(n=49)$, the rate of progression to probable DLB and probable AD was $20 \% \quad$ (20 per 100 person-years, 95\% CI 15.3-27.5) and $1.6 \%$ (1.6 per 100 person-years, 95\% CI 0.61-4.3), respectively [34]. Based on this clinical sample, it is therefore ten times more likely for amnestic MCI to progress to clinically probable AD than DLB, and the risk is ten times greater for non-amnestic MCI to progress to clinically probable DLB than AD.

\section{Survival and Mortality}

Of the eight identified studies reporting on survival and mortality outcomes in DLB $[29,35-41]$, six reported findings based on comparisons with AD $[29,35,36,39-41]$. Overall, when compared with patients with $\mathrm{AD}$, those with DLB reported worse results for various parameters measuring survivability and mortality, such as risk of hospital admission or death (reported as a single variable), survival time, and 5/10-year survival rates $[29,35,36,39-41]$.

For instance, a Japanese study reported that patients with DLB had a higher risk of hospital admission or death than those with AD (30\% vs $14 \% ; p<0.05$ ) [35]. A second Japanese study reported a lower 10-year survival rate for patients with DLB compared with $\mathrm{AD}$ and $\mathrm{VaD}$ (2.2\%, 18.9\%, and $13.2 \%$, respectively), albeit not significantly so, likely due to the small sample size in the DLB group [40]. Results from a Swedish study reported significantly shorter survival time from point of diagnosis and from point of achieving cognitive levels MMSE $20 \pm 1$ and MMSE $17 \pm 1$ (all $p<0.05$ ), and significantly lower 5-year survival rates in patients with DLB compared with those with
$\mathrm{AD}$ [36]. Additionally, a UK-based retrospective analysis of patient medical records reported "significantly" shorter median survival with cognitive impairment from first presentation in patients with DLB (3.72 years, 95\% CI 3.33-4.14) compared with those with AD (6.95 years, 95\% CI 5.78-8.12) [39]. Additionally, survival until CDR $=3$ or death was shown to be significantly shorter in a cohort of Norwegian patients with probable DLB compared with those with probable AD (1210 days and 1861 days, respectively; $p<0.0005$ ) [29]. In addition to the aforementioned clinical studies, a review study reported survival time estimates of 1.9-6.3 years after diagnosis with DLB versus 3.2-6.6 years after diagnosis with AD [41].

In a retrospective analysis of US patient records, the highest risk of death was observed in patients with multiple system atrophy (HR 7.85, 95\% CI 2.69-22.89, $p<0.001$ ), followed by PDD (HR 2.82, 95\% CI 1.8-4.4, $p<0.001$ ), DLB (HR 2.19, 95\% CI 2.15-4.73, $p<0.001$ ), and PD (HR 1.42, 95\% CI 1.13-1.78, $p=0.002$ ) $[37,38]$.

Predictors associated with survival were also reported in four identified studies [29, 41-43]. Multivariate analyses from a longitudinal prospective study in Sweden revealed that patients with DLB $(n=47)$ with elevated levels of cerebrospinal fluid total-tau ( $T$-tau) had increased risk of early death $(p=0.022$, HR 1.36 per $100 \mathrm{ng} / \mathrm{l} T$-tau, 95\% CI 1.05-1.78, model significance: $p=0.036$ ) [42]. This increased risk was not seen among patients with $\mathrm{AD}(n=157)$ or in the control group [42]. A Polish medical chart analysis of patients with AD and DLB from a university-based $\mathrm{AD}$ outpatient unit reported that mean survival time for DLB patients with diabetes was similar to the DLB group without diabetes (6.7 vs 6.3 years) [43]. In contrast, mean survival was significantly shorter for AD patients with diabetes than for the $\mathrm{AD}$ group without diabetes (6.3 vs 9.1 years) [43]. However, the authors concluded that the difference in mortality rates and survival time were not attributable to the presence of any vascular risk factor analysed [43]. A review looking into the prognosis of DLB reported key risk factors for earlier mortality, which included male gender, increased age at onset, increased burden of 
comorbidity, and functional impairment [41]. These factors are shared with patients with $\mathrm{AD}$, and can be considered generic mortality risk factors across the dementia spectrum [41]. Several specific predictors of shorter survival in patients with DLB as reported in the literature were also presented in this review, including gait abnormalities, fluctuating cognition, and hallucinations early in the disease course [41]. A Norwegian study found that statistically significant factors associated with shorter time to severe dementia $(C D R=3)$ or death included longer symptom duration, higher CDR global scores, and diagnosis with DLB (vs AD) [29]. These were already discussed within the "Cognitive decline" section of this review [29].

\section{Pharmaceutical Impact on Life Expectancy}

Due to the limited number of interventional studies, the impact of pharmaceutical intervention on mortality/survival in patients with DLB is unclear. In a single-clinic retrospective analysis of Japanese patients, life expectancy of 6.4 years and 3.6 years was reported in patients who received donepezil $(n=11)$ and those who did not $(n=28)$, respectively [44]. Although the authors concluded that this may have been due to a reduction in concomitant diseases, the publication was not explicit as to whether this reduction was due to donepezil.

In a separate study, a prospective, placebocontrolled, double-blinded trial was carried out to investigate the effect of memantine on survival in a pooled group of patients with DLB or PDD in Sweden $(n=75)$ [17]. This study itself was a continuation of a double-blinded 24 -week RCT conducted in Norway, UK, and Sweden between 2005 and 2008 [15]. Treatment response was recorded 24 weeks from baseline and measured by clinical global impression of change (CGIC). The 24-week RCT was followed by open-label treatment, and survival was recorded at 36 months [17]. Long-term data were available for 42 patients [17]. Kaplan-Meier estimates demonstrated that patients who received memantine during the first 24 weeks had higher 3-year survival than patients who received placebo $\left(\log \operatorname{rank} x^{2}=4.021, p=0.045\right)$ [17]. Additionally, patients that responded positively to memantine treatment after 24 weeks had a higher 3-year survival rate compared with non-responders (log rank $x^{2}=6.595, p=0.010$ ) [17]. However, due to the small sample size, multivariate Cox regression analysis to adjust for possible covariates with an effect on survival was not feasible [17].

\section{Clinical Management}

\section{Consensus Guidelines}

The DLB Consortium (DLBC), the European Federation of Neurological Societies-European Neurological Society (EFNS-ENS) Joint Congress of European Neurology, and the British Association for Psychopharmacology (BAP) have each issued detailed guidelines to assist clinicians in the management of patient with DLB (Table 1) [45-47]. However, of the three consensus guidelines identified in this review, only the DLBC criteria were developed with DLB as the primary focus and are therefore widely cited throughout the literature. The EFNS-ENS and BAP guidelines present recommendations aimed at patients with disorders associated with dementia in general; criteria specific to DLB are limited in these two sets of criteria.

In addition to the consensus guidelines, a number of other narrative review publications discussing the clinical management of DLB were identified as part of the current review (Table S8) [48-53].

\section{Diagnosis and Treatment Pathway}

\section{Clinical Diagnosis}

The consensus diagnosis criteria from the DLBC are summarised in Table 2. The DLBC distinguishes between clinical features and diagnostic biomarkers and provides guidance on how to establish and interpret these. Clinical signs and symptoms are weighted as core or supportive, and biomarkers as indicative or supportive, based upon their diagnostic specificity and the volume of good-quality evidence available. Although carrying less diagnostic weight, 
Table 2 Consensus diagnostic criteria from the DLBC. Adapted from McKeith et al. [46]

\section{Revised criteria for the clinical diagnosis of probable and possible DLB}

Essential for a diagnosis of DLB

Dementia, defined as a progressive cognitive decline of sufficient magnitude to interfere with normal social or occupational functions or with usual daily activities

Prominent or persistent memory impairment may not necessarily occur in the early stages but is usually evident with progression

Deficits on tests of attention, executive function, and visuoperceptual ability may be especially prominent and occur early

\section{Clinical features}

Core clinical features

Fluctuating cognition with pronounced variations in attention and alertness

Recurrent visual hallucinations that are typically well formed and detailed

REM sleep behaviour disorder, which may precede cognitive decline

One or more spontaneous cardinal features of parkinsonism

Bradykinesia (defined as slowness of movement and decrement in amplitude or speed)

Rest tremor

Rigidity

Note: the first three typically occur early and may persist throughout the course of disease

\section{Biomarkers}

Indicative biomarkers

Reduced dopamine transporter uptake in basal ganglia demonstrated by SPECT or PET

Abnormal (low uptake) ${ }^{123}$ iodine-MIBG myocardial scintigraphy

Polysomnographic confirmation of REM sleep without atonia

Diagnosis of DLB
Supportive clinical features

Severe sensitivity to antipsychotic agents

Postural instability

Repeated falls

Syncope or other transient episodes of unresponsiveness

Severe autonomic dysfunction (e.g., constipation, orthostatic hypotension, urinary incontinence)

Hypersomnia

Hyposmia

Hallucinations in other modalities

Systematised delusions

Apathy

Anxiety

Depression

Supportive biomarkers

Relative preservation of medial temporal lobe structures on CT/MRI scan

Generalised low uptake on SPECT/PET perfusion/metabolism scan with reduced occipital activity \pm the cingulate island sign on FDGPET imaging

Prominent posterior slow-wave activity on EEG with periodic fluctuations in the pre-alpha/theta range 
Table 2 continued

Revised criteria for the clinical diagnosis of probable and possible DLB

Probable DLB can be diagnosed if

(a) Two or more core clinical features of DLB are present, with or without the presence of indicative biomarkers, or

(b) Only one core clinical feature is present, but with one or more indicative biomarkers

Probable DLB should not be diagnosed on the basis of biomarkers alone

Possible DLB can be diagnosed if

(a) Only one core clinical feature of DLB is present, with no indicative biomarker evidence, or

(b) One or more indicative biomarkers are present but there are no core clinical features

DLB is less likely

(a) In the presence of any other physical illness or brain disorder, including cerebrovascular disease, sufficient to account in part or in total for the clinical picture, although these do not exclude a DLB diagnosis and may serve to indicate mixed or multiple pathologies contributing to the clinical presentation, or

(b) If parkinsonian features are the only core clinical feature and appear for the first time at a stage of severe dementia

Additional considerations

DLB should be diagnosed when dementia occurs before or concurrently with parkinsonism. The term PDD should be used to describe dementia that occurs in the context of well-established PD

In a practice setting, the term that is most appropriate to the clinical situation should be used, and generic terms such as Lewy body disease are often helpful

In research studies in which a distinction needs to be made between DLB and PDD, the existing 1-year rule between the onset of dementia and parkinsonism continues to be recommended

Produced by the authors for this publication based on data presented in McKeith et al. [46]

$C T$ computed tomography, $D L B$ dementia with Lewy bodies, DLBC Dementia with Lewy Bodies Consortium, EEG electroencephalogram, $F D G$ fluorodeoxyglucose, $M I B G$ metaiodobenzylguanidine, $M R I$ magnetic resonance imaging, $P D$ Parkinson's disease, $P D D$ Parkinson's disease dementia, $P E T$ positron emission tomography, REM rapid eye movement, SPECT single-photon emission computed tomography

supportive items are often valuable in clinical decision-making.

For the pathological assessment and diagnosis of DLB, previously published guidelines should continue to be used $[7,54]$. In the updated guidelines, only a few modifications were made to the criteria for assessing the likelihood that pathological findings are associated with DLB (Table S9) [46].

\section{Clinical Management}

Treatment of DLB is focused on the cognitive, psychiatric, motor, and other non-motor symptoms that represent the core features of the disorder [46]. Only supportive and palliative care can be offered to patients, as there are no medications available which will modify the course of disease. Treatment for DLB therefore remains symptomatic [45]. In general, DLB is treated with cautious use of anti-parkinsonian medication where necessary (levodopa monotherapy having the least proclivity to exacerbate psychosis) and acetylcholinesterase inhibitors (AChEIs) [45]. The marked loss of acetylcholine neurons in DLB forms the basis for AChEI use [50]. 
Table 3 Pharmacological and non-pharmacological approaches recommended in the DLBC consensus guidelines. Adapted from McKeith et al. [46]

\begin{tabular}{ll}
\hline Symptom type & Recommendations \\
\hline Non-pharmacological interventions \\
NA & Interventions can be patient- or caregiver-focused, or both \\
& Potential options based on preliminary evidence \\
& Exercise (both motor and cognitive benefits) \\
& Cognitive training \\
& Caregiver-oriented education and training to manage psychiatric symptoms including agitation \\
& and psychosis
\end{tabular}

Pharmacological interventions

Cognitive symptoms AChEIs

$\begin{array}{ll}\text { Neuropsychiatric } & \text { Memantine } \\ \text { symptoms } & \text { AChEIs } \\ & \text { Antipsychotics } \\ & \text { Drugs targeting serotonergic system }\end{array}$

Newer drugs targeting the serotonergic system, such as pimavanserin, may be alternatives; however, controlled clinical trial data in patients with DLB are needed

Although depressive symptoms are common in DLB, trial data are scant. In alignment with general advice on depression in dementia, SSRIs, SNRIs, and mirtazapine are options in DLB, with treatment guided by individual patient tolerability and response

Motor symptoms Levodopa

Safety assessments

Bone mineral density screening

Assessment of vitamin D status

Other symptoms A wide range of other symptoms can occur in DLB, including autonomic and sleep/wakefulness disturbances, which have profound negative sequelae for QoL in both patients and their families

In the absence of DLB-specific trial data for these symptoms, clinicians base their treatment decisions on clinical experience, expert opinion, or evidence-based recommendations developed in other diseases

For example, cautious bedtime use of clonazepam may reduce the risk of sleep-related injuries in patients with DLB with REM sleep behaviour disorder. However, it carries a risk of worsening cognition and gait impairment; melatonin represents a possibly safer option

$A C h E I$ acetylcholinesterase inhibitor, $D L B$ dementia with Lewy bodies, QoL quality of life, $D L B C$ The Dementia with Lewy Bodies Consortium, REM rapid eye movement, SNRI serotonin-norepinephrine reuptake inhibitor, SSRI selective serotonin reuptake inhibitor 
However, the management of DLB poses several challenges, as it requires balancing the treatment of a multitude of symptoms, including cognitive, neuropsychiatric, autonomic, and motor impairment. Treatment of one symptom often results in complications in other facets of the disease $[45,49]$. For example, dopamine replacement for motor symptoms may exacerbate neuropsychiatric symptoms, whereas antipsychotic treatment of hallucinations risks potentially fatal adverse reactions [49]. Additionally, AChEI treatment of cognitive symptoms may complicate cardiac and gastrointestinal dysautonomia [49]. It is because of these limitations that current recommendations are based, in part, upon consensus expert opinion [46]. Table 3 provides an overview of the non-pharmacological and pharmacological recommendations for the clinical management of patients with DLB, as recommended by the DLBC consensus guidelines [46].

\section{Hospitalisation and Cost of Care}

\section{Hospitalisation Rates and Duration}

Hospitalisation rates and duration in DLB patients were reported in three studies, one from the US and two based on the same UK cohort [55-57]. The US and UK cohorts stem from different regions and health systems and are therefore not comparable. Regarding generalisability, the UK cohort was derived from a real-life database, focusing on a specific geographic catchment of only four South London boroughs (Lambeth, Lewisham, Southwark, and Croydon) $[55,56]$. Although the reported data can be viewed as directional, they were very dependent on local care situations and discharge policies, which may not be comparable to other parts of London or elsewhere in the UK $[55,56]$. In comparison, details for the US cohort were limited in the identified abstract, which only indicated that these were DLB patients hospitalised at a university health network [57].

For the UK cohort, patients with DLB and AD were assembled from a large database of mental health and dementia care $[55,56]$. Overall, the rate of hospital admissions within the first year after dementia diagnosis was significantly higher in the 194 patients with DLB than in the 776 patients with $\mathrm{AD}$ (crude incidence rate ratio: $1.50,95 \%$ CI $1.28-1.75, p<0.001$ ) [56]. Mean follow-up time was 10.1 and 11.3 months for DLB and AD patients, respectively [56]. The mean number of planned admissions per person-year was higher in patients with DLB $(0.29$, 95\% CI 0.22-0.38) than in those with AD (0.20, 95\% CI 0.17-0.23) [56]. The mean number of unplanned admissions per person-year was also higher in patients with DLB $(1.02,95 \%$ CI $0.87-1.19)$ versus patients with $\mathrm{AD}(0.67,95 \%$ CI 0.61-0.73) [56]. Additionally, the mean number of hospital days per person-year in the year after diagnosis was significantly higher in patients with DLB $(10.8,95 \%$ CI 10.3-11.31) than in those with $\mathrm{AD}(6.92,95 \% \mathrm{CI} 6.73-7.11)$ [56]. The main driver of the increased rate of hospitalisation in patients with DLB was attributed to poorer physical health early in the disease course, whereas the length of stay appears to have been determined by neuropsychiatric symptoms [56]. Additionally, the rate of hospital admission was higher in the DLB group than in the general population (indirectly standardised hospitalisation rate $1.22,95 \%$ CI 1.06-1.39) [56].

The authors of this UK study released data for the same cohorts (albeit different numbers of patients: 160 patients with DLB and 640 patients with $\mathrm{AD}$ ) [55]. The overall findings were similar, with patients with DLB reporting significantly longer hospital stays than patients with AD (15.4 and 8.3 days/person-year, respectively) [55]. Patients with DLB also had significantly more hospital admissions than those with $\mathrm{AD}$ or the general older population in the catchment area (126.2, 93.5, and 87.5 per 100 person-years, respectively) [55]. Additionally, a strong association between DLB and hospitalisation was found in this cohort. This remained significant after adjusting for a wide range of confounders (HR 1.59, 95\% CI 1.19-2.13) [55].

Additional data were derived from a US cross-sectional study of DLB patients hospitalised at University of Florida Health [57]. A review of 179 hospital presentations found that the average length of hospitalisation was 
$7 \pm 8$ days [57]. After discharge, only 52\% returned to their previous living situation; the remaining $48 \%$ required a higher level of care [57]. This cross-sectional study also reported that the most common reasons for hospitalisation were worsened confusion or hallucinations (40\%), falls (24\%), and infection (23\%) [57].

\section{Cost of Care}

A 2008 cross-sectional study compared the total cost of care and its major components in a cohort of community-living patients from the US with probable $\mathrm{AD}(n=170)$ or (assumed confirmed) DLB $(n=25)$ [58]. The mean unadjusted total annual cost of care was higher in patients with DLB than in those with $A D$ $[\$ 35,143$ vs $\$ 25,129$ (constant 2004 USD), respectively], albeit not statistically significant [58]. Significantly higher indirect costs were reported for patients with DLB [\$23,036 vs $\$ 17,136$ (constant 2004 USD); $p<0.05$ ] [58]. Significantly lower direct non-medical costs were reported for patients with DLB than those with AD [\$947 vs \$1478 (constant 2004 USD); $p<0.01$ [ [58]. Lower direct non-medical costs were largely due to differences in home healthcare costs [ $\$ 739$ vs $\$ 1262$ for DLB and $\mathrm{AD}$, respectively (constant $2004 \mathrm{USD}$ ); $p<0.01$ ] [58]. However, It was not explicitly specified as to why DLB was associated with higher indirect costs than $\mathrm{AD}$, or what constituted these costs [58].

For the UK cohort discussed in the previous section, associated hospitalisation costs were determined by extracting publicly available healthcare resource group (HRG) data [56]. On average, patients with DLB incurred approximately $400 \mathrm{GBP} / 550$ USD more in hospitalisation costs (year of currency was not specified) than patients with $\mathrm{AD}$ in the first year after dementia diagnosis [56].

In a Swedish cross-sectional study, costs of care (year of currency was not specified) for patients with DLB were on average 348,000 Swedish Krona (SEK)/€37,500 per year compared with 169,000 SEK $(€ 18,200)$ in the AD group $(p<0.001)$ [59]. Care costs correlated significantly $\quad\left(r_{\mathrm{c}}=2.77, \quad p<0.001\right) \quad$ with dependency in instrumental activities of daily living (IADLs) measured with the Disability Assessment for Dementia (DAD). In contrast, MMSE and NPI status were not found to be significantly correlated with resource use [59].

In a separate study, the use and costs of formal care were estimated for the first 3 years after the diagnosis of mild dementia in patients in western Norway [60]. This study drew patients $(n=109)$ from a longitudinal incidence study of dementia, focusing on the four largest municipalities of the area (Bergen, Stavanger, Haugesund, or Sandnes) [60]. DLB-specific cost data were not reported [60]. However, important cost-predicting factors for patients with mild dementia were reported to be patients' living situation, being diagnosed with non-Alzheimer's disease, comorbidity, and daily living functioning [60].

In addition, one UK study assessed the costeffectiveness of AChEI treatment in patients with DLB $(n=112)$ and AD $(n=852)$ [61]. The cost per quality-adjusted life-year (QALY) gained with the use of AChEIs in patients with DLB was comparable to that for patients with moderate AD. Additionally, the cost-effectiveness of AChEIs was sufficient to recommend their use in routine practice [61].

\section{Quality of Life}

\section{QoL Instruments}

In total, six studies reporting on seven QoL instruments were identified as part of this review [62-67]. The AD-specific instruments ADRQL [62] and QOL-AD [63, 64] were utilised in one study and two studies, respectively. The PD-specific instrument PDQ-39 [65] and the dementia-specific instrument QOL-D [66] were reported in one study each. Two studies reported on one generic instrument each (EQ-5D [64] and the 12-Item Short Form Health Survey [SF12] [67]). The majority of publications reported results for multiple disease groups, including $\mathrm{AD}, \mathrm{VaD}, \mathrm{FTD}, \mathrm{PDD}, \mathrm{PD}, \mathrm{HD}$, and DLB. The only publication reporting on DLB exclusively assessed QoL using the PD-specific PDQ-39 instrument [65]. These findings highlight the lack of 
instrumentation specific to assessing QoL in DLB.

\section{QoL Outcomes}

One publication reported data only for patients with DLB, with results indicating that while mobility was the most impaired PDQ-39 domain, QoL had a stronger correlation with neuropsychiatric/behavioural features, e.g. depression, apathy, and ability to perform ADLs $(p<0.01)$, followed by anxiety $(p=0.02)$ [65].

Another study compared QoL between patients with DLB and patients with AD [64]. Based on AD-specific (QOL-AD) and generic (EQ-5D) instrument outcomes, patients with DLB reported worse patient- and caregiver-rated scores [64]. Two studies compared the QoL in patients with DLB against multiple disease groups [66, 67]. One study compared QoL between patients with $\mathrm{DLB}, \mathrm{AD}$, or FTD using the dementia-specific QOL-D instrument [66]. Of note, the apathy scores (measured via the 10-item NPI instrument) of FTD and DLB patients were significantly higher than those of patients with $\mathrm{AD}$, thereby affecting the lower positive affect (domain of the QOL-D instrument) of patients with FTD or DLB versus those with AD [66]. The second study compared QoL between patients with DLB, AD, or HD using the generic SF-12 instrument [67]. In this study, DLB was reported to be associated with worse physical health QoL than both $\mathrm{AD}$ and HD $(p<0.01)$ [67]. Overall, the results of these three studies indicate that patients with DLB have greater impairment of QoL than patients with other types of dementia, especially when compared with AD.

One study reported the QoL outcomes assessed by the ADRQL instrument in a mixed dementia group [62]. The results of multivariate analysis showed that behavioural and depressive symptoms of dementia patients, dependency in basic activities of daily living (BADL), poorer cognitive function, use of antipsychotic medication, caregiver burden, and caregiver not being an adult child were independent predictors of worse QoL in patients with dementia [62]. The remaining study compared QoL, via the QOL-AD instrument, between patients (DLB and PDD pooled) who received memantine and those who received placebo [63].

\section{Impact of Treatment}

In general, many types of commonly used medications can result in severe side effects in patients with DLB. Notably this includes medications with anticholinergic or antidopaminergic activity. Considerable improvement in QoL can be achieved by ceasing these treatments [48].

In a secondary analysis of an RCT involving 70 PDD and DLB patients, greater improvement in caregiver-rated QoL was seen for the QOL-AD item "life as a whole" in patients who received memantine versus placebo, with $42 \%$ and $15 \%$ rating QoL higher at 24 weeks in the memantine and placebo groups, respectively $(p=0.01)$ [63]. Additionally, significant improvements were seen in three out of seven caregiver domains [created by the authors by grouping QOL-AD items into domains based on the WHO International Classification of Functioning, Disability and Health (ICF)]: "total QOL" $(p=0.04)$, "body function" $(p=0.016)$, and "body structure" ( $p=0.047)$ [63].

\section{DISCUSSION}

The findings of this review indicate that patients with DLB typically reported worse outcomes in relation to efficacy and safety, cognitive decline, survival, and QoL. In addition, DLB was associated with higher hospitalisation rates and cost of care. Most of the findings were made in comparison to patients with AD.

However, the number of identified RCTs related to DLB was limited, reflecting the limited amount of clinical research in this field. Study duration, time points, patient demographics, and dosing varied widely among the identified studies. A high level of heterogeneity was also evident in the reported efficacy and safety outcomes. However, research (at various stages) is currently ongoing in assessing the efficacy and/or safety of novel treatments in patients with DLB (e.g. E2027, NPT200-11) 
$[68,69]$, signifying the increasing interest in fulfilling the unmet clinical need.

The management of DLB is difficult due to a lack of consensus on diagnostic and treatment criteria, which is reflected by the lack of consensus guidelines globally. This is further confounded by its similarities to other dementias. A potential solution for improving the management of DLB includes the establishment of national DLB networks, such as the DLB study group of the Italian Neurological Society for dementia (DLB-SINdem) [70]. Objectives of groups such as DLB-SINdem include improving the identification of DLB in dementia centres, particularly as the diagnosis of neurodegenerative dementia is often unbalanced towards $\mathrm{AD}$ in primary care [70]. This includes developing strategies to define and identify prodromal DLB [70], which is often misdiagnosed. Furthermore, national networks can contribute towards identifying available DLB cohorts, developing efficient data collection methodologies, and providing recommendations for conducting prospective cohort studies [70].

This review also concluded that hospitalisation is a major cost driver in dementia, particularly in patients with $\mathrm{AD}$, where the associated costs are well documented [55, 56, 58, 71-73]. However, despite DLB being the second most common type of neurodegenerative dementia, efforts to examine costs of care and the economic impact of treatment have been limited $[55,56,61]$. While there are several studies looking into the clinical and pathological aspects of DLB, studies around the economic aspect of neurodegenerative dementia have largely focused on $\mathrm{AD}$ [58]. Given the differences in rates of decline between patients with $\mathrm{DLB}$ and $\mathrm{AD}$, differences in disease costs over time are a possibility. Further work with longitudinal data is needed to assess the differences in disease cost trajectories.

A limitation of this review was the sole use of published data. Given that not all study data are published, it is possible that a degree of publication bias is present. Furthermore, a multitude of studies were published only in abstract form, thereby presenting limited data. Only publications reported in English were included as part of the literature review process, which may have inherently led to reporting bias, given that the only countries in which treatment for DLB is approved are predominantly non-Englishspeaking countries. Future research/reviews in this area would be enhanced by adding biomarkers and differential diagnosis to the search strategy.

Interest in the DLB has increased over the last few years. This is reflected by increased expenditure in this disease area, including the provision of a planning grant for a Europeanbased DLB consortium, which has led to several multi-site collaborations [74]. Additionally, in 2016, the National Institutes of Health (NIH) provided funding for the establishment of a nine-site US-based DLB consortium and several DLB-focused biomarker studies [74]. There is also increasing evidence of industry-based clinical trials and interest in the development of diagnostic tools focused on DLB [74].

\section{CONCLUSIONS}

Overall, the findings of this study show that patients with DLB exhibit worse outcomes in efficacy and safety, cognitive decline, survival, and QoL compared than do patients with AD. However, despite a number of studies looking into the clinical and pathological aspects of DLB, consensus guidelines and studies on healthcare utilisation in patients with dementia have largely focused on AD. Based on available information, only supportive and palliative care can be offered to patients, as there are no medications available which will modify the course of disease. Treatment for DLB therefore remains symptomatic. This limitation of data in the literature is an indication of the high unmet need associated with the area of therapy, and although clinical research in novel treatments for DLB is ongoing, it may be years before the clinical impact of these new candidates is realised. It is important, therefore, that raising awareness around DLB is prioritised in order to help inform patients, carers, and providers about the disease. 


\section{ACKNOWLEDGEMENTS}

Funding. This study and Rapid Service Fees for publication in Neurology and Therapy were funded by Eisai Inc.

Authorship. All named authors meet the International Committee of Medical Journal Editors (ICMJE) criteria for authorship for this manuscript, take responsibility for the integrity of the work as a whole, and have given final approval to the version to be published. All authors had full access to the articles reviewed in this manuscript and take complete responsibility for the integrity and accuracy of this manuscript.

Authorship Contributions. Amir Abbas Tahami Monfared and Genevieve Meier conceived of the study and were in charge of overall direction and planning and provided critical feedback to the other authors. Richard Perry and Darren Joe provided support to the design and implementation of the search strategy, analysis of data, and interpretation of the results.

Disclosures. Eisai employed Amir Abbas Tahami Monfared who played a significant part in study design, data collection, data analysis, data interpretation, and writing of the report. Eisai employed Genevieve Meier, who played a significant part in study design, data collection, data analysis, data interpretation, and writing of the report. Eisai provided funding to Richard Perry for their participation in the study. Eisai provided funding to Darren Joe for their participation in the study.

Compliance with Ethics Guidelines. This article is based on previously conducted studies and does not contain any studies with human participants or animals performed by any of the authors.

Open Access. This article is distributed under the terms of the Creative Commons Attribution-NonCommercial 4.0 International License (http://creativecommons.org/licenses/ by-nc/4.0/), which permits any noncommercial use, distribution, and reproduction in any medium, provided you give appropriate credit to the original author(s) and the source, provide a link to the Creative Commons license, and indicate if changes were made.

\section{REFERENCES}

1. Mayo Clinic. Lewy body dementia. 2019. https:// www.mayoclinic.org/diseases-conditions/lewybody-dementia/symptoms-causes/syc-20352025. Accessed Apr 2019.

2. Cromarty RA, Elder GJ, Graziadio S, et al. Neurophysiological biomarkers for Lewy body dementias. Clin Neurophysiol. 2016;127(1):349-59.

3. Seidel K, Mahlke J, Siswanto S, et al. The brainstem pathologies of Parkinson's disease and dementia with Lewy bodies. Brain Pathol. 2015;25(2):121-35.

4. Goedert M, Spillantini MG, Del Tredici K, Braak H. 100 years of Lewy pathology. Nat Rev Neurol. 2013;9(1):13.

5. Woodard JS. Concentric hyaline inclusion body formation in mental disease analysis of twentyseven cases. J Neuropathol Exp Neurol. 1962;21(3):442-9.

6. Kosaka K. Latest concept of Lewy body disease. Psychiatry Clin Neurosci. 2014;68(6):391-4.

7. McKeith IG, Galasko D, Kosaka K, et al. Consensus guidelines for the clinical and pathologic diagnosis of dementia with Lewy bodies (DLB) report of the consortium on DLB international workshop. Neurology. 1996;47(5):1113-24.

8. Papka M, Rubio A, Schiffer RB, Cox C. Lewy body disease: can we diagnose it? J Neuropsychiatry Clin Neurosci. 1998;10(4):405-12.

9. Lippa CF, Duda JE, Grossman M, et al. DLB and PDD boundary issues: diagnosis, treatment, molecular pathology, and biomarkers. Neurology. 2007;68(11):812-9.

10. Zweig YR, Galvin JE. Lewy body dementia: the impact on patients and caregivers. Alzheimers Res Ther. 2014;6(2):21.

11. Walker Z, Possin KL, Boeve BF, Aarsland D. Lewy body dementias. Lancet. 2015;386(10004):1683-97. 
12. Savica R, Grossardt BR, Bower JH, Boeve BF, Ahlskog JE, Rocca WA. Incidence of dementia with Lewy bodies and Parkinson disease dementia. JAMA Neurol. 2013;70(11):1396-402.

13. Lewy Body Dementia Association (LBDA). Lewy body dementia: the state of the science. 2016. https://www.lbda.org/sites/default/files/2016_lbd_ state_of_the_science_white_paper.pdf. Accessed 1 Apr 2019.

14. Higgins JPT, Green S, editors. Cochrane handbook for systematic reviews of interventions version 5.1.0. 2011. http://handbook.cochrane.org. Accessed 1 Apr 2019.

15. Aarsland D, Ballard C, Walker Z, et al. Memantine in patients with Parkinson's disease dementia or dementia with Lewy bodies: a double-blind, placebo-controlled, multicentre trial. Lancet Neurol. 2009;8(7):613-8.

16. Emre M, Tsolaki M, Bonuccelli U, et al. Memantine for patients with Parkinson's disease dementia or dementia with Lewy bodies: a randomised, doubleblind, placebo-controlled trial. Lancet Neurol. 2010;9(10):969-77.

17. Stubendorff K, Larsson V, Ballard C, Minthon L, Aarsland D, Londos E. Treatment effect of memantine on survival in dementia with Lewy bodies and Parkinson's disease with dementia: a prospective study. BMJ Open. 2014;4(7):e005158.

18. Wesnes KA, Aarsland D, Ballard C, Londos E. Memantine improves attention and verbal episodic memory in Parkinson's disease dementia and dementia with Lewy bodies: a double-blind, placebo-controlled multicentre trial. Alzheimers Dement. 2013;9(4):P890.

19. Wesnes KA, Aarsland D, Ballard C, Londos E. Memantine improves attention and episodic memory in Parkinson's disease dementia and dementia with Lewy bodies. Int J Geriatr Psychiatry. 2015;30(1):46-54.

20. ClinicalTrials.gov. A post-marketing clinical study of aricept in patients with dementia with Lewy bodies (DLB). 2018. https://clinicaltrials.gov/ct2/ show/NCT02345213. Accessed 1 Apr 2019.

21. Ikeda M, Mori E, Matsuo K, Nakagawa M, Kosaka K. Donepezil for dementia with Lewy bodies: a randomized, placebo-controlled, confirmatory phase III trial. Alzheimers Res Ther. 2015;7(1):4.

22. Mori E, Ikeda M, Nagai R, Matsuo K, Nakagawa M, Kosaka K. Long-term donepezil use for dementia with Lewy bodies: results from an open-label extension of phase III trial. Alzheimers Res Ther. $2015 ; 7(1): 5$.
23. Mori E, Ikeda M, Kosaka K, Donepezil-DLB Study Investigators. Donepezil for dementia with Lewy bodies: a randomized, placebo-controlled trial. Ann Neurol. 2012;72(1):41-52.

24. Cummings JL, Street J, Masterman D, Clark WS. Efficacy of olanzapine in the treatment of psychosis in dementia with lewy bodies. Dement Geriatr Cogn Disord. 2002;13(2):67-73.

25. Culo S, Mulsant BH, Rosen J, et al. Treating neuropsychiatric symptoms in dementia with Lewy bodies: a randomized controlled-trial. Alzheimer Dis Assoc Disord. 2010;24(4):360-4.

26. Wesnes KA, McKeith IG, Ferrara R, et al. Effects of rivastigmine on cognitive function in dementia with lewy bodies: a randomised placebo-controlled international study using the cognitive drug research computerised assessment system. Dement Geriatr Cogn Disord. 2002;13(3):183-92.

27. Kurlan R, Cummings J, Raman R, Thal L. Quetiapine for agitation or psychosis in patients with dementia and parkinsonism. Neurology. 2007;68(17):1356-63.

28. McKeith I, Del Ser T, Spano P, et al. Efficacy of rivastigmine in dementia with Lewy bodies: a randomised, double-blind, placebo-controlled international study. Lancet. 2000;356(9247):2031-6.

29. Rongve A, Soennesyn H, Skogseth R, et al. Cognitive decline in dementia with Lewy bodies: a 5-year prospective cohort study. BMJ Open. 2016;6(2):e010357.

30. Walker Z, McKeith I, Rodda J, et al. Comparison of cognitive decline between dementia with Lewy bodies and Alzheimer's disease: a cohort study. BMJ Open. 2012;2(1):e000380.

31. Kramberger MG, Auestad B, Garcia-Ptacek S, et al. Long-Term cognitive decline in dementia with lewy bodies in a large multicenter, international cohort. J Alzheimers Dis. 2017;57(3):787-95.

32. Smits LL, van Harten AC, Pijnenburg YA, et al. Trajectories of cognitive decline in different types of dementia. Psychol Med. 2015;45(5):1051-9.

33. Kramberger MG, Olmo JG, Lemstra AW, et al. Clinical characteristics and course of DLB: results from a large longitudinal multicentre cohort. Alzheimers Dement. 2015;11(7):P320.

34. Ferman TJ, Smith GE, Kantarci K, et al. Nonamnestic mild cognitive impairment progresses to dementia with Lewy bodies. Neurology. 2013;81(23):2032-8. 
35. Hanyu H, Sato T, Hirao K, Kanetaka H, Sakurai H, Iwamoto T. Differences in clinical course between dementia with Lewy bodies and Alzheimer's disease. Eur J Neurol. 2009;16(2):212-7.

36. Stubendorff K, Hansson O, Minthon L, Londos E. Differences in survival between patients with dementia with Lewy bodies and patients with Alzheimer's disease-measured from a fixed cognitive level. Dement Geriatr Cogn Disord. 2011;32(6):408-16.

37. Savica R, Ahlskog J, Boeve B, Bower J, Mielke M. Survival and risk of mortality of clinically-diagnosed synucleinopathies: a 15 year populationbased study (1991-2005) (P3. 350). Neurology. 2016;86(16 Supplement):P3. 350.

38. Savica R, Bower J, Hagen C, Eric A, Mielke M. Survival and risk of mortality of synucleinopathies: a 15 years population-based study (1991-2005). Mov Disord. 2016;31:S157.

39. Price A, Farooq R, Yuan J-M, Menon VB, Cardinal RN, O'Brien JT. Mortality in dementia with Lewy bodies compared with Alzheimer's dementia: a retrospective naturalistic cohort study. BMJ Open. 2017;7(11):e017504.

40. Matsui $\mathrm{Y}$, Tanizaki $\mathrm{Y}$, Arima $\mathrm{H}$, et al. Incidence and survival of dementia in a general population of Japanese elderly: the Hisayama study. J Neurol Neurosurg Psychiatry. 2009;80(4):366-70.

41. Mueller C, Ballard C, Corbett A, Aarsland D. The prognosis of dementia with Lewy bodies. Lancet Neurol. 2017;16(5):390-8.

42. Bostrom F, Hansson O, Blennow K, et al. Cerebrospinal fluid total tau is associated with shorter survival in dementia with Lewy bodies. Dement Geriatr Cogn Disord. 2009;28(4):314-9.

43. Magierski R, Kloszewska I, Sobow TM. The influence of vascular risk factors on the survival rate of patients with dementia with Lewy bodies and Alzheimer disease. Neurol Neurochir Pol. 2010;44(2):139-47.

44. Meguro K, Kato Y, Chida K, Nakamura K, Nakatsuka M, Yamaguchi S. Effect of drug treatment and nursing home residency on life expectancy in dementia (DLB vs $\mathrm{AD}$ ): a retrospective analysis in the Tajiri project. Alzheimers Dement. 2017;13(7):P385.

45. O'Brien JT, Holmes C, Jones M, et al. Clinical practice with anti-dementia drugs: a revised (third) consensus statement from the British Association for Psychopharmacology. J Psychopharmacol. 2017;31(2):147-68.
46. McKeith IG, Boeve BF, Dickson DW, et al. Diagnosis and management of dementia with Lewy bodies. Neurology. 2017;89(1):88-100.

47. Sorbi S, Hort J, Erkinjuntti T, et al. EFNS-ENS guidelines on the diagnosis and management of disorders associated with dementia. Eur J Neurol. 2012;19(9):1159-79.

48. Boot BP, McDade EM, McGinnis SM, Boeve BF. Treatment of dementia with lewy bodies. Curr Treat Opt Neurol. 2013;15(6):738-64.

49. Boot BP. Comprehensive treatment of dementia with Lewy bodies. Alzheimers Res Ther. 2015;7(1):45.

50. Gomperts SN. Lewy body dementias: dementia with Lewy bodies and Parkinson disease dementia. Continuum (Minneap Minn). 2016;22(2 Dementia):435.

51. Galasko D. Lewy body disorders. Neurol Clin 2017;35(2):325-38.

52. Latoo J, Jan F. Dementia with Lewy bodies: clinical review. Br J Gen Pract. 2008;1(1):10-4.

53. Macijauskienè J, Lesauskaitè V. Dementia with lewy bodies: the principles of diagnosis, treatment, and management. Medicina. 2012;48(1):1-8.

54. McKeith IG, Dickson DW, Lowe J, et al. Diagnosis and management of dementia with Lewy bodies: third report of the DLB Consortium. Neurology. 2005;65(12):1863-72.

55. Mueller C, Perera G, Rajamani A, et al. Risk and duration of hospitalization in dementia with Lewy bodies. Alzheimers Dement. 2017;13(7):P510-1.

56. Mueller C, Perera G, Rajkumar AP, et al. Hospitalization in people with dementia with Lewy bodies: frequency, duration, and cost implications. Alzheimers Dement (Amst). 2018;10:143-52.

57. Spears C, Besharat A, Martinez-Ramirez D, Almeida L, Armstrong M. Causes, complications and outcomes of hospitalization in patients with dementia with Lewy bodies [abstract]. Mov Disord 2017;32 (suppl 2). https://www.mdsabstracts.org/abstract/ causes-complications-and-outcomes-ofhospitalization-in-patients-with-dementia-with-lewy-bodies/. Accessed 6 Apr 2019.

58. Zhu CW, Scarmeas N, Stavitsky K, et al. Comparison of costs of care between patients with Alzheimer's disease and dementia with Lewy bodies. Alzheimers Dement. 2008;4(4):280-4.

59. Boström F, Jönsson L, Minthon L, Londos E. Patients with Lewy body dementia use more 
resources than those with Alzheimer's disease. Int J Geriatr Psychiatry. 2007;22(8):713-9.

60. Vossius C, Rongve A, Testad I, Wimo A, Aarsland D. The use and costs of formal care in newly diagnosed dementia: a three-year prospective follow-up study. Am J Geriatr Psychiatry. 2014;22(4):381-8.

61. Gustavsson A, Van Der Putt R, Jonsson L, McShane R. Economic evaluation of cholinesterase inhibitor therapy for dementia: comparison of Alzheimer's disease and dementia with Lewy bodies. Int J Geriatr Psychiatry. 2009;24(10):1072-8.

62. Mougias AA, Politis A, Lyketsos CG, Mavreas VG. Quality of life in dementia patients in Athens, Greece: predictive factors and the role of caregiverrelated factors. Int Psychogeriatr. 2011;23(3):395-403.

63. Larsson V, Engedal K, Aarsland D, Wattmo C, Minthon L, Londos E. Quality of life and the effect of memantine in dementia with Lewy bodies and Parkinson's disease dementia. Dement Geriatr Cogn Disord. 2011;32(4):227-34.

64. Bostrom F, Jonsson L, Minthon L, Londos E. Patients with dementia with Lewy bodies have more impaired quality of life than patients with Alzheimer disease. Alzheimer Dis Assoc Disord. 2007;21(2):150-4.

65. Armstrong M, Monari E, Almeida L, McFarland N, Malaty I, Okun M. Initial patterns and correlates of quality of life in dementia with Lewy bodies (P6. 234). Neurology. 2016;86(16 Supplement):P6. 234.

66. Kurisu K, Terada S, Oshima E, et al. Comparison of QOL between patients with different degenerative dementias, focusing especially on positive and negative affect. Int Psychogeriatr. 2016;28(8):1355-61.
67. Figari-Jordan R, Anderson K, Gruber-Baldini A, et al. Comparison of quality of life and disability in three different dementias (P07. 180). Neurology. 2012;78(1 Supplement):P07. 180.

68. ClinicalTrals.gov. Study to evaluate the efficacy, safety and tolerability of E2027 in subjects with dementia with Lewy bodies. 2018. https:// ClinicalTrials.gov/show/NCT03467152. Accessed 1 Apr 2019.

69. Price DL, Koike MA, Khan A, et al. The small molecule alpha-synuclein misfolding inhibitor, NPT200-11, produces multiple benefits in an animal model of Parkinson's disease. Sci Rep. 2018;8(1):16165.

70. Bonanni L, Cagnin A, Agosta F, et al. The Italian dementia with Lewy bodies study group (DLB-SINdem): toward a standardization of clinical procedures and multicenter cohort studies design. Neurol Sci. 2017;38(1):83-91.

71. Zhao Y, Kuo TC, Weir S, et al. Healthcare costs and utilization for medicare beneficiaries with Alzheimer's. BMC Health Serv Res. 2008;8:108.

72. Taipale H, Purhonen M, Tolppanen AM, et al. Hospital care and drug costs from five years before until two years after the diagnosis of Alzheimer's disease in a Finnish nationwide cohort. Scand J Public Health. 2016;44(2):150-8.

73. Phelan EA, Borson S, Grothaus L, Balch S, Larson EB. Association of incident dementia with hospitalizations. JAMA. 2012;307(2):165-72.

74. Leverenz JB. Global initiatives and future challenges. Alzheimers Dement. 2017;13(7):P1445. 